\title{
Gastrointestinal Tumors
}

This section provides information for researchers and health care professionals who are interested in enrolling patients into currently recruiting clinical trials. Studies may be funded by the NIH, other federal agencies, nonprofit organizations, or industry. Listed are only phase II/III trials currently recruiting in Europe that are registered in the ClinicalTrials.gov database.

In this issue of ONCOLOGY Research AND TREATMENT trials on gastrointestinal tumors are listed.

\author{
Randomized Controlled Trial to Prevent Peritoneal Seeding in Gastric Cancer (HIPEC_Stomach) \\ Condition: Gastric cancer, peritoneal carcinomatosis ClinicalTrials.gov identifier: NCT01683864 Phase: II/III \\ Intervention: Drug: HIPEC with mytomycin and cisplatin; procedure: diagnostic laparoscopy one year after gastrectomy \\ Cytoreductive Surgery (CRS) with/without HIPEC in Gastric Cancer with Peritoneal Carcinomatosis (GASTRIPEC) \\ Condition: Malignant neoplasm of stomach, secondary malignant ClinicalTrials.gov identifier: NCT02158988 Phase: III \\ neoplasm of peritoneum \\ Intervention: Procedure: HIPEC
}

A Study of Ramucirumab (LY3009806) in Combination with Capecitabine and Cisplatin in Participants with Stomach Cancer (RAINFALL)

Condition: Metastatic gastric adenocarcinoma, gastroesophageal ClinicalTrials.gov identifier: NCT02314117 Phase: III

Intervention: Drug: ramucirumab; drug: capecitabine; drug: cisplatin; drug: placebo; drug: fluorouracil

A Study of Ramucirumab (LY3009806) in Combination with Paclitaxel in Participants with Gastric Cancer

Condition: Gastric adenocarcinoma, gastroesophageal junction ClinicalTrials.govidentifier: NCT02514551 Phase: I

adenocarcinoma
Intervention: Drug: ramucirumab; drug: paclitaxel

A Study of BBI608 Plus Weekly Paclitaxel to Treat Gastric and Gastro-Esophageal Junction Cancer (BRIGHTER)

Condition: Gastric cancer, gastroesophageal junction cancer ClinicalTrials.gov identifier: NCT02178956 Phase: III

Intervention: Drug: BBI608; drug: paclitaxel; other: placebo

HELOISE Study: A Study of Herceptin (Trastuzumab) in Combination with Cisplatin/Capecitabine Chemotherapy in Patients with HER2-Positive Metastatic Gastric or Gastro-Esophageal Junction Cancer

Condition: Gastric Cancer ClinicalTrials.gov identifier: NCT01450696 Phase: III

Intervention: Drug: capecitabine; drug: cisplatin; drug: trastuzumab

A Study of Pembrolizumab (MK-3475) Versus Paclitaxel for Participants with Advanced Gastric/Gastroesophageal Junction Adenocarcinoma that Progressed after Therapy with Platinum and Fluoropyrimidine (MK-3475-061/KEYNOTE-061)

Condition: Gastric adenocarcinoma; gastroesophageal junction ClinicalTrials.gov identifier: NCT02370498 Phase: III

Intervention: Biological: pembrolizumab; drug: paclitaxel

Study of Pembrolizumab (MK-3475) as First-Line Monotherapy and Combination Therapy for Treatment of Advanced Gastric or Gastroesophageal Junction Adenocarcinoma (MK-3475-062/KEYNOTE-062)

Condition: Gastric adenocarcinoma, gastroesophageal junction ClinicalTrials.gov identifier: NCT02494583 Phase: III

$\begin{array}{ll}\text { adenocarcinoma } & \\ \text { Intervention: } \quad \text { Biological: pembroliziumab; drug: cisplatin + 5-fluorouracil, cisplatin + 5-fluorouracil + placebo }\end{array}$

Study of Pembrolizumab (MK-3475) in Previously-Treated Participants with Advanced Carcinoma of the Esophagus or Esophagogastric Junction (MK-3475-180/KEYNOTE-180)

Condition: Esophageal cancer ClinicalTrials.gov identifier: NCT02559687 Phase: II

Intervention: Biological: pembroliziumab

Study of Pembrolizumab (MK-3475) Versus Investigator's Choice Standard Therapy for Participants with Advanced Esophageal/Esophagogastric Junction Carcinoma that Progressed after First-Line Therapy (MK-3475-181/KEYNOTE-181)

Condition: Esophageal cancer ClinicalTrials.govidentifier: NCT02564263 Phase: III

Intervention: Biological: pembroliziumab; investigator's choice: paclitaxel or docetaxel or irinotecan 
Definitive Radiochemotherapy Plus/Minus Cetuximab in Unresectable Locally Advanced Esophageal Cancer

Condition: Esophageal Cancer ClinicalTrials.gov identifier:

Intervention: Drug: cetuximab; drug: cisplatin, 5-FU; radiation: radiotherapy

ClinicalTrials.gov identifier: NCT01787006 Phase: II

Evaluation for the Individualization of Therapy in Adenocarcinomas of the Gastroesophageal Junction (MEMORI)

Condition: Adenocarcinoma of the esophagogastric junction ClinicalTrials.govidentifier: NCT02287129 Phase: II

Intervention: Drug: oxaliplatin; drug: epirubicin; drug: capecitabine; drug: 5-FU; drug: carboplatin; drug: paclitaxel; radiation: radiation; procedure: biopsy

Integration of Trastuzumab, with or without Pertuzumab, into Perioperative Chemotherapy of HER2-Positive Stomach Cancer: The INNOVATION Trial (EORTC-1203-GITCG)

$\begin{array}{ll}\text { Condition: } & \begin{array}{l}\text { Resectable, HER2-positive gastric or gastroesophageal ClinicalTrials.gov identifier: NCT02205047 } \\ \text { junction adenocarcinoma (UICC stage Ib-III) }\end{array} \\ \text { Intervention: } & \text { Drug: cisplatin; drug: 5-fluorouracil or capecitabine; drug: trastuzumab; drug: pertuzumab; procedure: gastrectomy }\end{array}$

FLO +/- Pazopanib as First-line Treatment in Advanced Gastric Cancer (PaFLO)

$\begin{array}{llll}\text { Condition: } & \text { Advanced gastric cancer } & \text { ClinicalTrials.gov identifier: NCT01503372 Phase: } & \text { II } \\ \text { Intervention: } & \text { Drug: pazopanib; drug: 5-FU, oxaliplatin, leukovorin (FLO) } & \end{array}$

Intervention: Drug: pazopanib; drug: 5-FU, oxaliplatin, leukovorin (FLO)

Preoperative Chemosensitivity Testing to Predict Treatment Benefit in Adjuvant Stage III Colon Cancer (PePiTA)

Condition: Colon cancer ClinicalTrials.govidentifier: NCT00994864 Phase: III

Intervention: Drug: FOLFOX

Regorafenib in Subjects with Metastatic Colorectal Cancer (CRC) Who Have Progressed after Standard Therapy (CONSIGN)

Condition: Colon cancer ClinicalTrials.govidentifier: NCT01538680 Phase: III

Intervention: Drug: regorafenib (BAY73-4506)

Phase 3 Study to Evaluate Efficacy and Safety of Masitinib in Comparison to Sunitinib in Patients with Gastrointestinal Stromal Tumour after Progression with Imatinib

Condition:

NCT01694277

Phase:

III

Intervention: Drug: masitinib, drug: sunitinib

Study of Pembrolizumab (MK-3475) as Monotherapy in Participants with Previously-Treated Locally Advanced Unresectable or Metastatic Colorectal Cancer (MK-3475-164/KEYNOTE-164)
Condition:
Colorectal cancer
ClinicalTrials.gov identifier:
NCT02460198
Phase: II
Intervention: Biological: pembrolizumab

Study of Pembrolizumab (MK-3475) vs Standard Therapy in Participants with Microsatellite Instability-High (MSI-H) or Mismatch Repair Deficient (dMMR) Stage IV Colorectal Carcinoma (MK-3475-177/KEYNOTE-177)
Condition:
Colorectal cancer
ClinicalTrials.gov identifier:
NCT02563002
Phase:
III
Intervention: Biological: pembrolizumab; investigator's choice: mFOLFOX6 or mFOLFOX6 + bevacizumab or mFOLFOX6+ cetuximab or FOLFIRI or FOLFIRI+ bevacizumab or FOLFIRI+ cetuximab

Evaluation of MGN1703 Maintenance Treatment in Patients with mCRC with Tumor Reduction During Induction Treatment (IMPALA)

Condition: Metastatic colorectal cancer

ClinicalTrials.gov identifier:

NCT02077868 Phase:

III

Intervention: Other: usual maintenance, drug: MGN1703 treatment

Cap+Bev vs Cap+Iri+Bev 1st-line Therapy in mCRC

Condition: Colorectal cancer, metastatic

Intervention: Drug: capecitabine, drug: bevacizumab, drug: irinotecan

ClinicalTrials.gov identifier: NCT01249638 Phase: III

Effects of Deep Regional Hyperthermia in Patients with Anal Carcinoma Treated by Standard Radiochemotherapy (HYCAN)

Intervention: Radiation: irradiation; drug: mitomycin C; drug: 5-fluorouracil; procedure: hyperthermia

Endoscopic Stenting Versus Surgical Bypass for Low Bile Duct Obstruction by Cancer of the Pancreatic Head (STENTBY)

$\begin{array}{llll}\text { Condition: } & \text { Pancreatic cancer ClinicalTrials.gov identifier: NCT00753441 Phase: II/III } \\ \text { Intervention: } & \text { Procedure: surgical bypass; procedure: endoscopic stenting }\end{array}$

Pancreatic Carcinoma: Chemoradiation Compared with Chemotherapy Alone after Induction Chemotherapy (CONKO-007)
Condition:
Pancreatic cancer
ClinicalTrials.gov identifier:
NCT01827553
Phase:
III

Intervention: Drug: induction chemotherapy with gemcitabine or FOLFIRINOX; radiation: radiotherapy, 28 x 1.8 Gy; drug: chemotherapy, gemcitabine; drug: chemotherapy with gemcitabine or FOLFIRINOX according to induction chemotherapy

Neoadjuvant Treatment in Resectable Pancreatic Cancer (NEOPA)

Condition: Pancreatic cancer ClinicalTrials.govidentifier: NCT01900327 Phase: III

Intervention: Radiation: external beam radiation; drug: gemcitabine neoadjuvant; procedure: surgery; drug: gemcitabine adjuvant 
Randomized Multicenter Phase II/III Study with Adjuvant Gemcitabine Versus Neoadjuvant / Adjuvant FOLFIRINOX for Resectable Pancreas Carcinoma

Condition: Resectable prancreas carcinoma ClinicalTrials.govidentifier: NCT02172976 Phase: II/II

Intervention: Drug: gemcitabine; drug: oxaliplatin; drug: 5-fluorouracil; drug: irinotecan; drug: natriumfolinate

Hyperthermia European Adjuvant Trial (HEAT)

Condition: Resected pancreatic adenocarcinoma ClinicalTrials.govidentifier: NCT01077427 Phase: III

Intervention: Device: gemcitabine + cisplatin + regional hyperthermia; drug: gemcitabine

Efficacy and Safety of Everolimus and (STZ-5FU) Given One Upfront the Other Upon Progression in Advanced pNET (SEQTOR)

Condition: Pancreatic neuroendocrine tumours ClinicalTrials.gov identifier: NCT02246127 Phase: III

Intervention: Drug: everolimus; drug: STZ-5FU

Survivin Peptide Vaccination for Patients with Advanced Melanoma, Pancreatic, Colon and Cervical Cancer

ClinicalTrials.gov identifier:

NCT00108875 Phase: I/II

Intervention: Biological: survivin peptide vaccine

Study of Cabozantinib (XL184) vs Placebo in Subjects with Hepatocellular Carcinoma Who Have Received Prior Sorafenib (CELESTIAL)

Intervention: Drug: cabozantinib tablets; drug: placebo tablets

ClinicalTrials.gov identifier: NCT01908426 Phase: III

A Study of Ramucirumab (LY3009806) Versus Placebo in Participants with Hepatocellular Carcinoma and Elevated Baseline Alpha-Fetoprotein (REACH-2

Condition: Hepatocellular carcinoma ClinicalTrials.govidentifier: NCT02435433 Phase: II

Intervention: Drug: ramucirumab; drug: placebo

Study of ThermoDox with Standardized Radiofrequency Ablation (RFA) for Treatment of Hepatocellular Carcinoma (HCC) (OPTIMA)

Condition: Hepatocellular carcinoma ClinicalTrials.govidentifier: NCT02112656 Phase: III

Intervention: Drug: ThermoDox; drug: dummy infusion

Efficacy and Safety Doxorubicin Transdrug Study in Patients Suffering From Advanced Hepatocellular Carcinoma (ReLive)

Condition: Hepatocellular carcinoma ClinicalTrials.gov identifier: NCT01655693 Phase: III

Intervention: Drug: doxorubicin; drug: best standard of care

Study of Regorafenib after Sorafenib in Patients with Hepatocellular Carcinoma (RESORCE)

Condition: Hepatocellular carcinoma ClinicalTrials.gov identifier: NCT01774344 Phase: III

Intervention: Drug: regorafenib (BAY73-4506); drug: placebo

Efficacy Evaluation of TheraSphere in Patients with Inoperable Liver Cancer (STOP-HCC)

$\begin{array}{lllll}\text { Condition: } & \text { Unresectable hepatocellular carcinoma } & \text { ClinicalTrials.gov identifier: NCT01556490 Phase: III } \\ \text { Intervention: } & \text { Device: TheraSphere } & \end{array}$ 